\title{
REWRITING SYSTEMS AND EMBEDDING OF MONOIDS IN GROUPS
}

\author{
FABIENNE CHOURAQUI
}

\begin{abstract}
In this paper, a connection between rewriting systems and embedding of monoids in groups is found. We show that if a group with a positive presentation has a complete rewriting system $\Re$ that satisfies the condition that each rule in $\Re$ with positive lefthand side has a positive right-hand side, then the monoid presented by the subset of positive rules from $\Re$ embeds in the group. As an example, we give a simple proof that right angled Artin monoids embed in the corresponding right angled Artin groups. This is a special case of the well-known result of Paris [11] that Artin monoids embed in their groups.
\end{abstract}

\section{INTRODUCTION}

In [1], Adian gave a sufficient condition on the presentation of a monoid for its embeddability in a group, which is very simple to check.

Adian's results have been extended in different directions in the works of several authors, for example, in the paper of Kashintsev [8] where various small cancellation conditions are used to study embeddability of semigroups in groups and in the paper of Krstic [9].

In this work, instead looking at the presentation of the monoid, we consider the group presentation. In section 2, we give some preliminaries on embeddability of monoids in groups and on rewriting systems. In section 3 , we give the main result and we give some definitions. We define a positive word in the group $G=\operatorname{Gp}\langle X \mid R\rangle$ to be a word which belongs to the free monoid $X^{*}$ and we consider the empty word " 1 " as a positive word. We say that a rewriting system $\Re$ for the group $G=\operatorname{Gp}\langle X \mid R\rangle$ satisfies the condition $C^{+}$if each rule in $\Re$ with positive left-hand side has a positive right-hand side. We define $\Re^{+}$to be the subset of all rules of $\Re$ with positive left-hand side. If $\Re$ satisfies the condition $C^{+}$then the rules in $\Re^{+}$are called positive rules. 
The main result proved in section 3 can then be stated as follows:

Theorem. Let $N$ be a monoid and $G=\operatorname{Gp}\langle X \mid R\rangle$ a group. Assume that $G$ has a complete rewriting system $\Re$ that satisfies the condition $C^{+}$. Then the monoid $M=\operatorname{Mon}\left\langle X \mid \Re^{+}\right\rangle$embeds into $G$ as the monoid of positive words relative to this presentation. In particular, if $N$ is isomorphic to $M$, then $N$ embeds into $G$.

Using this result, in section 4 , we give a simple and natural proof that right angled Artin monoids embed in their corresponding right angled Artin groups. This is a special case of the well-known result of Paris [11] that Artin monoids embed in their groups. If $G$ is a right angled Artin group, then we define for $G$ a terminating rewriting system $\Re_{0}$, that is based on a length-lexicographical ordering induced by a total ordering on the generators, which satisfies the condition $C^{+}$and such that $\Re_{0}^{+}$ is a rewriting system for the monoid with the same presentation. We then apply the Knuth-Bendix algorithm of completion on $\Re_{0}$ in order to obtain a complete (maybe infinite) rewriting system $\Re$, which is equivalent to $\Re_{0}$. We then show that the rewriting system $\Re$ satisfies the condition $C^{+}$and we show that at each step of the completion a positive rule is obtained from an ambiguity between positive rules which were obtained at a former step.

This gives an algorithm for checking the embeddability of a monoid $M$ generated by a set $X$ into a group $G=\operatorname{Gp}\langle X \mid R\rangle$ which can be described in the following way: Define for $G$ a terminating rewriting system $\Re_{0}$, which is based on a total ordering of the words, that satisfies the condition $C^{+}$and such that $\Re_{0}^{+}$is a rewriting system for $M$.

Apply the Knuth-Bendix algorithm of completion on $\Re_{0}$ in order to obtain a complete rewriting system $\Re$, which is equivalent to $\Re_{0}$.

If the complete rewriting system $\Re$ does not satisfy the condition $C^{+}$, then nothing can be concluded.

If the complete rewriting system $\Re$ does satisfy the condition $C^{+}$and at each step a positive rule is created by an ambiguity between positive rules which were created at a former step, then $M$ embeds in $G$.

Acknowledgment. This work is a part of my Ph-d thesis, done at the Technion under the supervision of Professor Arye Juhasz. I am very grateful to Professor Arye Juhasz, for his patience, his encouragement and his many judicious remarks and to the referee for his very useful comments. 


\section{Preliminaries}

2.1. On embedding of monoids in groups. Let $\Sigma$ be a non-empty set. We denote by $\Sigma^{*}$ the free monoid generated by $\Sigma$; elements of $\Sigma^{*}$ are finite sequences called words and the empty word will be denoted by 1 . Let $M$ be a monoid. The left and right laws of cancellation hold in $M$ if the following conditions are satisfied: if $a b=a c$ implies $b=c$ and $b a=c a$ implies $b=c$ respectively. A commutative monoid with laws of cancellation can always be embedded in a group. This is not necessarily true for non-commutative monoids, so for this class of monoids the question if there is a group $G$ such that $M$ can be embedded in $G$ arises. Adian, in [1], gives a partial answer to this question: he gives a sufficient condition for embeddability of the monoid in the group with the same presentation. In what follows, we will describe Adian's criteria for embeddability. Let $M$ be a monoid with presentation $\left\langle\Sigma \mid r_{1}, r_{2}, . ., r_{m}\right\rangle$, where each relation $r_{i}$ have the form $u_{i}=v_{i}, u_{i}, v_{i} \in \Sigma^{*}$ for $1 \leq i \leq m$, and none of the words $u_{i}, v_{i}$ is empty. Adian in [1] defined for such a presentation the following two graphs, which are called the left and right graphs of the presentation. In the left graph, $\Gamma_{l}$, to each relation $r_{i}$ there corresponds an edge $\left(a_{i}^{1}, b_{i}^{1}\right)$, where $a_{i}^{1}$ is the first letter of the word $u_{i}$ and $b_{i}^{1}$ is the first letter of the word $v_{i}$. In the right graph, $\Gamma_{r}$, to each relation $r_{i}$ there corresponds an edge $\left(a_{i}, b_{i}\right)$, where $a_{i}$ is the last letter of the word $u_{i}$ and $b_{i}$ is the last letter of the word $v_{i}$. A sequence of edges $\left(a_{1}, a_{2}\right),\left(a_{2}, a_{3}\right), . .,\left(a_{k-1}, a_{k}\right)$ is called a path connecting $a_{1}$ and $a_{k}$ and if $a_{1}=a_{k}$ then this path is closed. A closed path of length greater than 1 such that the first and last edges of the path are distinct, i.e. correspond to different relations and all vertices $a_{j}$, for $1<j<k$, are different from $a_{1}$ is called a cycle. A closed path of length 1 is an elementary cycle.

Theorem 2.1. [1, p.57] Let $M$ be a monoid with presentation $\left\langle\Sigma \mid r_{1}, r_{2}, . ., r_{m}\right\rangle$, where each relation $r_{i}$ have the form $u_{i}=v_{i}, u_{i}, v_{i} \in$ $\Sigma^{*}$ for $1 \leq i \leq m$, and none of the words $u_{i}, v_{i}$ is empty. Assume there are no cycles in the left and right graphs of the presentation of $M$ and let $w_{1}, w_{2}$ be words in $M$. Then the equality $w_{1}=w_{2}$ holds in the monoid $M$ if and only if it holds in the group $G$ presented in terms of the same generators and the same defining relations and in this case $M$ embeds in $G$.

\section{Examples}

1. Let $M=\langle a, b \mid a b=b a\rangle$. The left and right graphs $\Gamma_{l}$ and $\Gamma_{r}$ are the same and none of them has any cycle, so the monoid embeds in the free abelian group on two generators. 
2. Let $M=\langle x, y \mid x y=y\rangle$. There is no left cycle but there is an elementary right cycle (from $y$ to itself). That means that the monoid is left cancellative but not necessarily right cancellative.

\subsection{On rewriting systems.}

We refer the reader to [2], [5] and [7] for more details.

Definition. A rewriting system $\Re$ on $\Sigma$ is a set of ordered pairs in $\Sigma^{*} \times \Sigma^{*}$.

If $(l, r) \in \Re$ then for any words $u$ and $v$ in $\Sigma^{*}$, we say the word $u l v$ reduces to the word urv and we write $u l v \rightarrow u r v$. A word $w$ is said to be reducible if there is a word $z$ such that $w \rightarrow z$. If there is no such $z$ we call $w$ irreducible.

A rewriting system $\Re$ is called terminating (or Noetherian) if there is no infinite sequence of reductions $w_{1} \rightarrow w_{2} \rightarrow \ldots \rightarrow w_{n} \rightarrow \ldots$

We denote by " $\rightarrow$ " the reflexive transitive closure of the relation " $\rightarrow$ ".

A rewriting system $\Re$ is called Church - Rosser if for any words $u, v$ in $\Sigma^{*}, u \leftrightarrow^{*} v$ implies that there is a word $z$ in $\Sigma^{*}$ such that $u \rightarrow^{*} z$ and $v \rightarrow^{*} z$ (i.e. if $u$ and $v$ are equivalent then they have a common descendant). $\Re$ is called confluent if for any words $u, v, w$ in $\Sigma^{*}$, $w \rightarrow^{*} u$ and $w \rightarrow^{*} v$ implies that there is a word $z$ in $\Sigma^{*}$ such that $u \rightarrow^{*} z$ and $v \rightarrow^{*} z$ (i.e. if $u$ and $v$ have a common ancestor then they have a common descendant). These two properties of $\Re$ are equivalent: $\Re$ is Church-Rosser if and only if $\Re$ is confluent 2]. For any word $w$ in $\Sigma^{*}$, the confluence of $\Re$ ensures the existence of at most one irreducible equivalent word.

$\Re$ is called locally confluent if for any words $u, v, w$ in $\Sigma^{*}, w \rightarrow u$ and $w \rightarrow v$ implies that there is a word $z$ in $\Sigma^{*}$ such that $u \rightarrow^{*} z$ and $v \rightarrow^{*} z$.

$\Re$ is called complete (or convergent) if $\Re$ is terminating and confluent. So, if $\Re$ is complete then every word $w$ in $\Sigma^{*}$ has a unique irreducible equivalent word $z$ and $z$ is called the normal form of $w$.

It is a very hard task to determine the completeness of an arbitrary rewriting system. Knuth and Bendix have elaborated an algorithm which for a given finite and terminating rewriting system $\Re$, tests its completeness and if $\Re$ is not complete then new rules are added to complete it. Instead of testing the confluence of $\Re$, the algorithm tests the locally confluence of $\Re$, since for a terminating rewriting system $\Re$ locally confluence and confluence are equivalent [2, p.16].

We say that two rewriting systems $\Re$ and $\Re^{\prime}$ are equivalent if : $w_{1} \leftrightarrow^{*}$ $w_{2}$ modulo $\Re$ if and only if $w_{1} \leftrightarrow^{*} w_{2}$ modulo $\Re^{\prime}$. So, by applying the Knuth-Bendix algorithm on a terminating rewriting system $\Re$ a 
complete rewriting system $\Re^{\prime}$ which is equivalent to $\Re$ can be found. If the algorithm halts after a finite number of steps and the rewriting system obtained $\Re^{\prime}$ is complete then $\Re^{\prime}$ is finite and complete, since a finite number of rules are added.

We call the triple of non-empty words $u, v, w$ in $\Sigma^{*}$ an overlap ambiguity if there are $r_{1}, r_{2}$ in $\Sigma^{*}$ such that $u v \rightarrow r_{1}$ and $v w \rightarrow r_{2}$ are rules in $\Re$. We then say that $r_{1} w$ and $u r_{2}$ are the corresponding critical pair. When the triple $u, v, w$ in $\Sigma^{*}$ is an overlap ambiguity we will say that the rules $u v \rightarrow r_{1}$ and $v w \rightarrow r_{2}$ overlap at $v$ or that there is an overlap between the rules $u v \rightarrow r_{1}$ and $v w \rightarrow r_{2}$. If there exists a word $z$ such that $r_{1} w \rightarrow^{*} z$ and $u r_{2} \rightarrow^{*} z$, then we say that the critical pair resulting from the overlap of the rules $u v \rightarrow r_{1}$ and $v w \rightarrow r_{2}$ in $\Re$ resolves.

The triple $u, v, w$ of possibly empty words in $\Sigma^{*}$ is called an inclusion ambiguity if there are $r_{1}, r_{2}$ in $\Sigma^{*}$ (which must be distinct if both $u$ and $w$ are empty, but otherwise may be equal) such that $v \rightarrow r_{1}$ and $u v w \rightarrow$ $r_{2}$ are rules in $\Re$. We then say that $u r_{1} w$ and $r_{2}$ are the corresponding critical pair. If there exists a word $z$ such that $u r_{1} w \rightarrow^{*} z$ and $r_{2} \rightarrow^{*} z$, then we say that the critical pair resulting from the inclusion ambiguity of the rule $v \rightarrow r_{1}$ in $u v w \rightarrow r_{2}$ in $\Re$ resolves. Given a terminating rewriting system $\Re$, the Knuth-Bendix algorithm may halt after a finite number of steps and fail if the words in a critical pair cannot be compared in order to resolve it. If a total ordering of the words is defined, then the algorithm ensures the existence of a complete rewriting system $\Re^{\prime}$ which is equivalent to $\Re$ (see [7]).

So, $\Re$ is complete if $\Re$ is terminating and locally confluent or in other words if $\Re$ is terminating and all the critical pairs resolve.

\section{The MAIn Result: A CRITERIA FOR EMBEDDABILITy OF MONOIDS IN GROUPS}

In this section, we use the following notation.

Let $M$ be a monoid and let $G$ be the group presented by $\operatorname{Gp}\langle X \mid R\rangle$, so the monoid presentation of $G$ is $\operatorname{Mon}\left\langle X \cup X^{-1} \mid R \cup R_{0}\right\rangle$, where $X^{-1}$ denotes the set $\left\{x_{0}^{-1}, \ldots, x_{n}^{-1}\right\}$ and $R_{0}=\left\{x_{i} x_{i}^{-1}=1, x_{i}^{-1} x_{i}=1\right.$, with $i \in\{0,1, \ldots, n\}\}$. We refer the reader to [13] for more details.

Definition 3.1. Let $w$ be a word in $\left(X \cup X^{-1}\right)^{*}$. We say that $w$ is a positive word in $G$ if $w$ belongs to the free monoid $X^{*}$. The empty word " 1 " is considered here as a positive word.

Example: The braid group $B_{3}$ with the presentation $B_{3}=\operatorname{Gp}\langle a, b, c|$ $\left.a^{3}=b^{2}=c\right\rangle$.

The word $a c$ is a positive word in $B_{3}$, while the word $a^{-1} c$ is not. 
Definition 3.2. We denote by $\Re^{+}$the subset of all rules of $\Re$ with positive left-hand side. We allow in $\Re^{+}$rules of the form $l \rightarrow 1$, where $l$ is a positive word.

Definition 3.3. Let $\Re$ be a rewriting system for $G$, with $\Re^{+} \neq \emptyset$. We say that $\Re$ satisfies the condition $C^{+}$if each rule in $\Re$ with positive left-hand side has a positive right-hand side.

Example: In [12], the authors give a finite and complete rewriting system for the group $B_{3}$, which is described as follows:

$\Re=\left\{a^{-1} \rightarrow c^{-1} a^{2}, b^{-1} \rightarrow c^{-1} b, a^{3} \rightarrow c, b^{2} \rightarrow c, a c \rightarrow c a, a c^{-1} \rightarrow\right.$ $\left.c^{-1} a, b c \rightarrow c b, b c^{-1} \rightarrow c^{-1} b, c c^{-1} \rightarrow 1, c^{-1} c \rightarrow 1\right\}$

The rewriting system $\Re$ satisfies the condition $C^{+}$, since each rule in $\Re$ with positive left-hand side has a positive right-hand side. The rewriting system $\Re^{+}$is: $\Re^{+}=\left\{a^{3} \rightarrow c, b^{2} \rightarrow c, a c \rightarrow c a, b c \rightarrow c b\right\}$

Remark 3.4. One can check that the rewriting system $\Re^{+}$described above is a finite and complete rewriting system for the monoid presented by $\left\langle a, b, c \mid a^{3}=b^{2}=c\right\rangle$.

Definition 3.5. We say that $\Re^{+}$is a rewriting system for $M$ if $M$ is isomorphic to the factor monoid $X^{*} / \equiv_{\Re^{+}}$.

Lemma 3.6. Assume that $G$ has a complete rewriting system $\Re$ which satisfies the condition $C^{+}$. If $\Re^{+}$is a rewriting system for $M$, then $\Re^{+}$ is a complete rewriting system for $M$. Further, if $\Re$ is finite then $\Re^{+}$ is also finite.

Proof. We have to show that $\Re^{+}$is terminating and that all the critical pairs resulting from any kind of ambiguity between rules in $\Re^{+}$resolve by using rules in $\Re^{+}$.

Assume there is an infinite sequence of reductions in $\Re^{+}$: $w_{1} \rightarrow w_{2} \rightarrow \ldots w_{m} \rightarrow . . \rightarrow$..

Since the rules applied in this sequence are rules in $\Re^{+}$, these are also rules in $\Re$. So, there is an infinite sequence of reductions in $\Re$. But this is a contradiction to the fact that $\Re$ is terminating. So, $\Re^{+}$is terminating.

Assume that $u$ and $v$ is a critical pair resulting from any kind of ambiguity between rules in $\Re^{+}$. Since $\Re$ is complete, this critical pair resolves using rules in $\Re$. But the words $u$ and $v$ are positive words, since $\Re$ satisfies the condition $C^{+}$. So, the rules from $\Re$ used to resolve this critical pair belong to $\Re^{+}$. So, $\Re^{+}$is complete. If $\Re$ is finite, then $\Re$ is also finite, since $\Re^{+}$is a subset of $\Re$.

Definition 3.7. Let $\Re$ be a rewriting system. We denote by $\equiv_{\Re}$ the equivalence relation generated by $\Re$. In fact, $\equiv_{\Re}$ is a congruence since it is compatible with concatenation. (see [13]) 
Lemma 3.8. Assume that $G$ has a complete rewriting system $\Re$ which satisfies the condition $C^{+}$. Let $u, v$ be positive words. Then $u \equiv_{\Re} v$ if and only if $u \equiv_{\Re^{+}} v$.

Proof. If $u \equiv_{\Re^{+}} v$, then clearly $u \equiv_{\Re} v$, since $\Re^{+} \subseteq \Re$.

Assume that $u \equiv_{\Re} v$. Then there is a (modulo $\Re$ ) irreducible word $z$ such that $u \rightarrow^{*} z$ and $v \rightarrow^{*} z$. The words $u$ and $v$ are positive words in $G$ and $\Re$ satisfies the condition $C^{+}$, so the rules used in order to reduce $u$ and $v$ to $z$ belong to $\Re^{+}$and $z$ is also a positive word. So, $u \equiv_{\Re^{+}} v$.

Theorem 3.9. Let $N$ be a monoid and $G=\operatorname{Gp}\langle X \mid R\rangle$ a group. Assume that $G$ has a complete rewriting system $\Re$ that satisfies the condition $C^{+}$. Then the monoid $M=\operatorname{Mon}\left\langle X \mid \Re^{+}\right\rangle$embeds into $G$ as the monoid of positive words relative to this presentation. In particular, if $N$ is isomorphic to $M$, then $N$ embeds into $G$.

Proof. Assume that $\Re^{+}$is a rewriting system for $M$. Then from lemma 3.6, $\Re^{+}$is a complete rewriting system for $M$. Let $u$ and $v$ be positive words such that $u$ and $v$ are equal in $G$. We show that the words $u$ and $v$ are equal in $M$. Since $\Re$ is a complete rewriting system for $G, u$ and $v$ are equal in $G$ implies that $u \equiv_{\Re} v$. From lemma 3.8, $u \equiv_{\Re^{+}} v$ since $u$ and $v$ are positive words. But $\Re^{+}$is a complete rewriting system for $M$, so $u$ and $v$ are equal in $M$.

\section{An Application:Embedding of Right Angled Artin MONOIDS}

Recall that a group $\mathrm{G}$ is called an Artin group and the corresponding monoid is called an Artin monoid if it is presented by a set $X=$ $\left\{x_{1}, \ldots, x_{n}\right\}$ subject to relations of the form $\underbrace{x_{i} x_{j} x_{i} . .}_{m_{i, j}}=\underbrace{x_{j} x_{i} x_{j} .}_{m_{j, i}}$ where $m_{i, j}=m_{j, i} \geq 2$ or $m_{i, j}=0$ in which case we omit the relation between $x_{i}$ and $x_{j}$. A right-angled Artin group (or partially commutative group) is one in which $m_{i, j} \in\{2,0\}$. In other words, in the presentation for the Artin group, all relations are commutator relations: $x_{i} x_{j}=x_{j} x_{i}$. A standard way to specify the presentation for any Artin group is by means of the defining graph $\Im$. Let $\Im$ be a finite, simplicial graph with edges labeled by integers greater than one. The Artin group (resp. the Artin monoid) associated to $\Im$ is the group (resp. monoid) whose presentation has generators corresponding to the vertices $x_{1}, . ., x_{n}$ and there is a relation $\underbrace{x_{i} x_{j} x_{i} .}_{m_{i, j}}=\underbrace{x_{j} x_{i} x_{j} . .}_{m_{i, j}}$ for each edge labeled $m_{i, j}$ connecting $x_{i}$ and $x_{j}$. Several authors have studied the question of embeddability of general Artin monoids in Artin groups: for example special 
cases of this question have been considered by Charney [3] and Cho and Pride [4]. Paris [11] proved the following general result about Artin groups and Artin monoids: Every Artin monoid embeds in the corresponding Artin group.

In this section, we show the following theorem.

Theorem 4.1. Right angled Artin monoids embed in their corresponding groups.

The proof is by showing that they admit a complete rewriting system $\Re$ satisfying the condition $C^{+}$and the condition on $\Re^{+}$.

In [6] Hermiller and Meier constructed finite and complete rewriting systems for graph groups, and as a special case, for right angled Artin groups using another presentation. In [14], Van Wyk constructs complete rewriting systems for graph groups. His main interest is on the normal forms. Although we obtain the same normal forms as in [14], our main interest is on the rewriting system and on how the rules look like in the light of theorem 3.9 .

Let $\Im$ be the defining graph of a right-angled Artin group $G$. Denote by $X$ the set of vertices in $\Im$ (or equivalently the set of generators of $G$ ) and by $X^{-1}$ the set of inverses of the elements in $X$.

Lemma 4.2. To each edge $a-b$ in $\Im$, there corresponds the following set of 4 relations in the monoid presentation of the group: $\left\{a^{\epsilon} b^{\delta}=b^{\delta} a^{\epsilon}\right.$, where $\epsilon, \delta$ take all values \pm 1$\}$

Proof. To each edge $a-b$ in $\Im$, there corresponds the relation $a b=b a$ in the group presentation. In the monoid presentation of the group, by adding the relations: $x x^{-1}=1$ and $x^{-1} x=1$ for every $x \in X$, we obtain a set with many relations which are all consequences of the set of 4 relations described above.

Lemma 4.3. Each set of 4 relations corresponding to an edge $a-b$ in $\Im$, as defined in lemma 4.2, can be oriented in two ways such that the overlaps with the rules $x x^{-1} \rightarrow 1$ and $x^{-1} x \rightarrow 1(x \in X)$ resolve: $\left\{a^{\epsilon} b^{\delta} \rightarrow b^{\delta} a^{\epsilon}\right.$, where $\epsilon, \delta$ take all values \pm 1$\}$ or $\left\{b^{\delta} a^{\epsilon} \rightarrow a^{\epsilon} b^{\delta}\right.$, where $\epsilon, \delta$ take all values \pm 1$\}$

Remark 4.4. Note that in both cases, when the left-hand side of a rule is a positive word its right-hand side is also a positive word.

Proof. We will check that the overlap of the first rule (in the first set) with a rule of free reduction resolve and we omit this technical checking for the other rules.

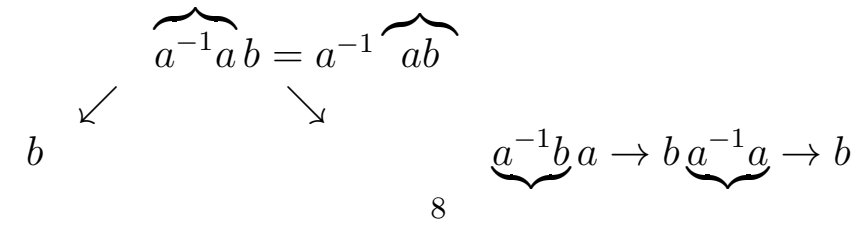


From lemma 4.3, we have that for any right angled Artin group $G$, by defining a total ordering on the generators and using the lengthlexicographical ordering induced by it, one can define a rewriting system of the following form: $\Re_{0}=\left\{x^{\gamma} y^{\epsilon} \rightarrow y^{\epsilon} x^{\gamma}\right.$, where $\gamma$ and $\epsilon$ take all values \pm 1 , for each edge $x-y \in \Im\}$. If $\Re_{0}$ is finite and complete with no need of completion, then it satisfies the condition $C^{+}$(see remark 4.4) and $\Re_{0}^{+}$is a rewriting system for $M$. Yet, this is not true in general, an example of infinite complete rewriting system is given in [6, p.250]. We will show here (propositions 4.9] and 4.10) that by applying the Knuth-Bendix algorithm of completion on $\Re_{0}$, an equivalent rewriting system (not necessarily finite) $\Re$ is obtained which is complete and which satisfies the condition $C^{+}$and the condition on $\Re^{+}$. Since $\Re_{0}$ is terminating and there is a total ordering of the words, the algorithm ensures the existence of an equivalent rewriting system $\Re$ which is complete. This will imply that the monoid embeds in the corresponding group, by theorem 3.9 ,

Remark 4.5. In order to avoid inclusion ambiguities, we will use the following strategy of reduction in the Knuth-Bendix algorithm of completion: at each step, we reduce each word in the critical pair to its normal form modulo the rewriting system obtained at the earlier step. So, we need consider only overlap ambiguities.

Before we proceed, we need the following definitions and notations.

Let $w=t_{1} t_{2} \ldots t_{k}$, where $t_{i} \in X \bigcup X^{-1}$ for $1 \leq i \leq k$.

We define the prefix of $w$ to be the following set of words:

$\operatorname{pref}(w)=\left\{t_{1}, t_{1} t_{2}, t_{1} t_{2} t_{3}, . ., t_{1} t_{2} t_{3} \ldots t_{k}\right\}$

We denote by $\ell(w)$ the length of the word $w$.

We denote by $\Re$ the complete rewriting system obtained by applying the Knuth-Bendix algorithm of completion on $\Re_{0}$ and we denote by $\Re_{n}$ the rewriting system obtained at the $n$-th step in the algorithm of completion, that is $\Re_{n}=\Re_{n-1} \bigcup\{$ the rules obtained at the $n$-th step\}. We use the following definition from [14]: the set $\left\{t_{1}, t_{2}, . ., t_{k}\right\}$ is defined to be a clique if for all $1 \leq i, j \leq k, i \neq j, t_{i}$ and $t_{j}$ commute.

Lemma 4.6. Assume the following set of rules occur in $\Re_{0}$ :

$$
(*)\left\{\begin{aligned}
t_{1} x & \rightarrow x t_{1} \\
x t_{i} & \rightarrow t_{i} x
\end{aligned}\right.
$$

where $t_{1}, t_{i}, x \in X \bigcup X^{-1}$ for $2 \leq i \leq k$ and such that $\left\{t_{1}, t_{2}, . ., t_{k}, x\right\}$ is not a clique.

Assume there is a rule $u x \rightarrow x u$ in $\Re$, where $u=t_{1} t_{i_{2}} . . t_{i_{k}}$ and $t_{i_{j}} \in\left\{t_{2}, . ., t_{k}\right\}$. Then

(i) the following rules also occur in $\Re: u^{\prime} x \rightarrow x u^{\prime}$ for every $u^{\prime} \in \operatorname{pref}(u)$. 
Further, these rules are obtained at a step earlier than $u x \rightarrow x u$.

(ii) the following rule also occurs in $\Re: u x^{-1} \rightarrow x^{-1} u$

The proof is technical and appears in appendix A. The main idea is that we apply step by step the algorithm of completion on the rules in $\left(^{*}\right)$ and the existence of the rule $u x \rightarrow x u$ in $\Re$ ensures the creation of the rules $u^{\prime} x \rightarrow x u^{\prime}$ for every $u^{\prime} \in \operatorname{pref}(u)$ and the rule $u x^{-1} \rightarrow x^{-1} u$.

For brevity of notation, we say that the rule $u x \rightarrow x u$ satisfies the condition on prefixes if $u=t_{1} t_{2} . . t_{k}$, where $x, t_{i} \in X \bigcup X^{-1}$ and the rules $t_{1} x \rightarrow x t_{1}$ and $x t_{i} \rightarrow t_{i} x$ for $2 \leq i \leq k$ occur in $\Re_{0}$.

Definition 4.7. Let $u v \rightarrow u^{\prime}$ and $v w \rightarrow v^{\prime}$ be rules in a rewriting system $\Re$, where $v$ is not the empty word. Then we say that there is an overlap between these rules with $u v \rightarrow u^{\prime}$ at left and $v w \rightarrow v^{\prime}$ at right. We call $v$ the overlapping word and $\ell(v)$ the length of the overlap.

In the following lemma, we show that the length of the overlaps in $\Re$ cannot exceed one, that is the overlapping words belong to $X \bigcup X^{-1}$.

Lemma 4.8. Assume the rules $u x_{1} x_{2} . . x_{m} \rightarrow x_{m} u x_{1} x_{2} . . x_{m-1}$ and $x_{1} x_{2} . . x_{m} y \rightarrow y x_{1} x_{2} . . x_{m}$ satisfy the condition on prefixes and occur in $\Re$. Then $m=1$.

Proof. Assume $m>1$. The rule $u x_{1} x_{2} . . x_{m} \rightarrow x_{m} u x_{1} x_{2} . . x_{m-1}$ satisfies the condition on prefixes, so the rule $x_{m} x_{1} \rightarrow x_{1} x_{m}$ occurs in $\Re_{0}$, that is $\left(^{*}\right) x_{m}>x_{1}$. The rule $x_{1} x_{2} . . x_{m} y \rightarrow y x_{1} x_{2} . . x_{m}$ satisfies the condition on prefixes, so the rule $y x_{m} \rightarrow x_{m} y$ occurs in $\Re_{0}$, that is $x_{1}>y$ and $y>x_{m}$. So, we have $x_{1}>y>x_{m}$. But, this contradicts $(*)$, so $m=1$.

Proposition 4.9. (i) The equivalent complete rewriting system $\Re$ obtained from the Knuth-Bendix algorithm of completion of $\Re_{0}$ satisfies the following conditions:

(A) All the rules in $\Re$ have the following form $u x \rightarrow x u$, where $u=t_{1} t_{2} . . t_{k-1} t_{k}$ is a word, $x, t_{i} \in X \bigcup X^{-1}$, and they satisfy the condition on prefixes.

(B) Only overlaps between rules from $\Re$ at left and rules from $\Re_{0}$ at right produce new rules, that is all the other kinds of overlaps resolve.

(C)At the $n$-th step of completion, the rules produced have words of length $n+2$ in each side.

(ii) $\Re$ satisfies the following condition: one side of a rule in $\Re$ is a positive word if and only if the other side is a positive word. In particular, $\Re$ satisfies the condition $C^{+}$(even if it is not finite). 
Proof. (i) The proof will be by induction on the number of steps in the Knuth-Bendix algorithm of completion of $\Re_{0}$. We show by induction that $\Re$ satisfies the conditions (A), (B) and (C).

At the 0 -th step, for each rule of kind $x^{\gamma} y^{\epsilon} \rightarrow y^{\epsilon} x^{\gamma}$ in $\Re_{0}$, where $\gamma, \epsilon= \pm 1$, assumption (A) holds trivially. Each rule has words of length 2 in each side.

At the first step, we have overlaps between rules $x^{\gamma} y^{\epsilon} \rightarrow y^{\epsilon} x^{\gamma}$ and $y^{\epsilon} z^{\delta} \rightarrow z^{\delta} y^{\epsilon}$, where $\gamma, \delta, \epsilon= \pm 1$, and a new rule is obtained: $x^{\gamma} z^{\delta} y^{\epsilon} \rightarrow$ $y^{\epsilon} x^{\gamma} z^{\delta}$. So, assumptions (A) and (C) hold.

We need to check that the assumption (B) holds also:

Let $y^{\epsilon} z^{\prime \beta} \rightarrow z^{\prime \beta} y^{\epsilon}$ and $z^{\prime \beta} x^{\prime \alpha} \rightarrow x^{\prime \alpha} z^{\prime \beta}$, where $\alpha, \beta, \epsilon= \pm 1$, be rules in $\Re_{0}$ whose overlap produced the rule: $y^{\epsilon} x^{\prime \alpha} z^{\prime \beta} \rightarrow z^{\prime \beta} y^{\epsilon} x^{\prime \alpha}$. We need to show that the overlap of $x^{\gamma} z^{\delta} y^{\epsilon} \rightarrow y^{\epsilon} x^{\gamma} z^{\delta}$ at left with $y^{\epsilon} x^{\prime \alpha} z^{\prime \beta} \rightarrow$ $z^{\prime \beta} y^{\epsilon} x^{\prime \alpha}$ at right resolve. But, there is an overlap of $x^{\gamma} z^{\delta} y^{\epsilon} \rightarrow y^{\epsilon} x^{\gamma} z^{\delta}$ at left with $y^{\epsilon} z^{\prime \beta} \rightarrow z^{\prime \beta} y^{\epsilon}$ at right which created the following rule: $x^{\gamma} z^{\delta} z^{\prime \beta} y^{\epsilon} \rightarrow y^{\epsilon} x^{\gamma} z^{\delta} z^{\prime \beta}$.

So, we have that the overlap resolves (which is denoted by $\checkmark$ ):

$$
\underbrace{\overbrace{x^{\gamma} z^{\delta} y^{\epsilon}} x^{\prime \alpha} z^{\prime \beta}}_{y^{\epsilon} x^{\gamma} z^{\delta} x^{\prime \alpha} z^{\prime \beta}} \underbrace{\searrow \underbrace{x^{\gamma} z^{\delta} z^{\prime \beta} y^{\epsilon}}} x^{\prime \alpha} \rightarrow y^{\epsilon} x^{\gamma} z^{\delta} \underbrace{z^{\prime \beta} x^{\prime \alpha}}
$$

So, at the first step the assumption (B) is satisfied.

Assume that at the $(n-1)$-th step of the Knuth-Bendix algorithm of completion, each new rule obtained satisfies the induction assumptions (A), (B) and (C). At the $n$-th step, a new rule is obtained from an overlap ambiguity between a rule obtained at the $(n-1)$-th step and a rule obtained at the $(n-1)$-th step or at earlier steps. Let $u x \rightarrow x u$ be a rule obtained at the $(n-1)$-th step which satisfies the condition on prefixes, where $u=t_{1} t_{2} . . t_{k-1} t_{k}$ and $x, t_{i} \in X \bigcup X^{-1}$. Assume the rule $x v y \rightarrow y x v$ in $\Re_{n-1}$ satisfies the condition on prefixes, where $v$ is a word and $y \in X \bigcup X^{-1}$.

If $v$ is not the empty word, then by the induction assumption (B) the overlap between $u x \rightarrow x u$ and $x v y \rightarrow y x v$ resolve. So, let $v$ be the empty word, that is we consider the overlap between the rules $u x \rightarrow x u$ and $x y \rightarrow y x$ :

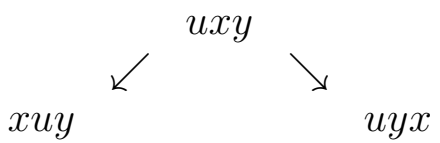

There are three cases to check. We show first that in each case conditions (A) and (C) are satisfied.

First case: If $x u y$ and $u y x$ are freely reduced and irreducible modulo 
$\Re_{n-1}$, then a new rule is created: $u y x \rightarrow x u y$ which satisfies the condition on prefixes, that is the assumption (A) holds in $\Re_{n}$. Since by the induction assumption $(\mathrm{C}) \ell(u x)=\ell(x u)=(n-1)+2=n+1$, we have that $\ell(u y x)=\ell(x u y)=(n+1)+1=n+2$, so $(\mathrm{C})$ holds.

Second case: Assume $x u y$ and $u y x$ are freely reduced but may be reducible modulo $\Re_{n-1}$.

If there is a rule $u y \rightarrow y u$ in $\Re_{n-1}$, then the overlap resolves, by using first the rule $u y \rightarrow y u$ and then the rules $u x \rightarrow x u$ and $x y \rightarrow y x$.

If $u=u_{1} u_{2}$, where $u_{1}$ is a non-empty word, and $u_{2} y \rightarrow y u_{2}$ occurs in $\Re_{n-1}$, then we have:

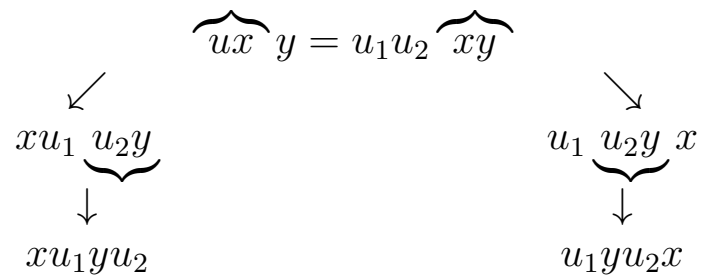

So, a new rule is created: $u_{1} y u_{2} x \rightarrow x u_{1} y u_{2}$ which satisfies the condition on prefixes, so the conditions (A) and (C) are satisfied.

Third case: Assume $x u y$ and $u y x$ are not freely reduced, then $u y$ is not freely reduced that is $t_{k}=y^{-1}$, since all the subwords $x u, u$ and $y x$ are reduced.

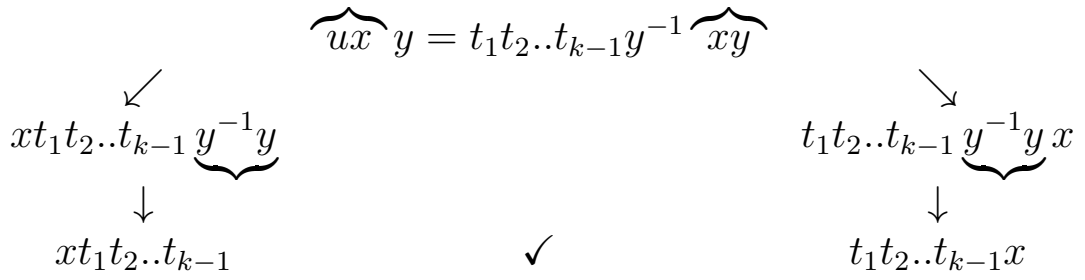

The word $t_{1} t_{2} . . t_{k-1}$ belongs to $\operatorname{pref}(u)$ and the rule $u x \rightarrow x u$ in $\Re_{n-1}$ satisfies the condition on prefixes, so by lemma 4.6, there is a rule $t_{1} t_{2} . . t_{k-1} x \rightarrow x t_{1} t_{2} . . t_{k-1}$ which was obtained at an earlier step. So, this overlap resolves using this rule.

We have shown that the conditions (A) and (C) hold in $\Re$. Now, we need to show that the condition (B) holds too.

Let $u x \rightarrow x u$ be a rule in $\Re_{n}$ which satisfies the condition on prefixes. Assume $x v y \rightarrow y x v$ is a rule in $\Re_{n} \backslash \Re_{0}$, where $v=s_{1} s_{2} . . s_{k}$ and $s_{i} \in X \cup X^{-1}$, such that there is an overlap with $u x \rightarrow x u$ at left and $x v y \rightarrow y x v$ at right. In order to show that the condition (B) holds, we need to check that this overlap resolves.

From assumption (A), $x v y \rightarrow y x v$ satisfies also the condition on prefixes. Then: 


$$
\begin{aligned}
& \overbrace{u x} v y=u \overbrace{x s_{1} s_{2} . . s_{k} y} \\
& x u v y=\stackrel{\swarrow}{x u s_{1} s_{2} . . s_{k} y} \\
& u y x v=u y x s_{1} s_{2} . . s_{k}
\end{aligned}
$$

Since $x v y \rightarrow y x v$ satisfies the condition on prefixes, there are rules $x y \rightarrow y x$ and $y s_{i} \rightarrow s_{i} y$ for $1 \leq i \leq k$ in $\Re_{0}$. So, there is an overlap of the rule $u x \rightarrow x u$ at left and $x y \rightarrow y x$ at right, which created the rule $u y x \rightarrow x u y$. So, we have using this rule and the rules $y s_{i} \rightarrow s_{i} y$, for $1 \leq i \leq k$ that:

$$
\overbrace{u x}^{u y=u \overbrace{\substack{x s_{1} s_{2} . . s_{k} y \\ x u v y}} \quad \checkmark \underbrace{u y x} s_{1} . . s_{k} \rightarrow x u \underbrace{y s_{1}} . . s_{k} \rightarrow . . \rightarrow x u s_{1} . . s_{k} y}
$$

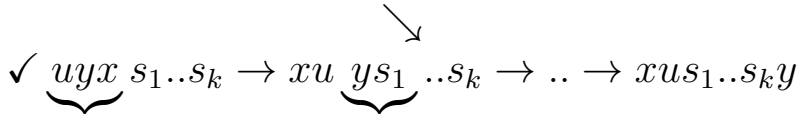

So, this overlap resolves. At last, we need to check that the overlaps of rules with rules of free reduction resolve. Let $u x \rightarrow x u$ be a rule which satisfies the condition on prefixes, where $u=t_{1} t_{2} . . t_{k-1} t_{k}$ and $x, t_{i} \in X \bigcup X^{-1}$. We check first the overlap with $u x \rightarrow x u$ at left and $x x^{-1} \rightarrow 1$ at right:

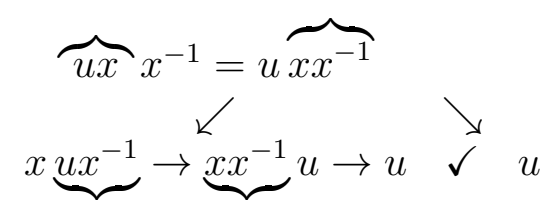

We check now the overlap with $t_{1}^{-1} t_{1} \rightarrow 1$ at left and $u x \rightarrow x u$ at right.

$$
\begin{aligned}
\overbrace{t_{1}^{-1} t_{1}} t_{2} . . t_{k-1} t_{k} x=t_{1}^{-1} \overbrace{t_{1} t_{2} . . t_{k-1} t_{k} x} & \\
\swarrow & \checkmark \underbrace{t_{2} . . t_{k-1}^{-1} t_{k} x} u \rightarrow x \underbrace{t_{1}^{-1} u} \stackrel{\searrow}{\rightarrow} \underbrace{x t_{2}} . . t_{k-1} t_{k} \rightarrow . .
\end{aligned}
$$

(ii) From (i), all the rules in $\Re$ have the form $u x \rightarrow x u$. So, the word $u x$ is positive if and only if the word $x u$ is positive.

Proposition 4.10. Let $\Re$ be the complete rewriting system obtained from the Knuth-Bendix algorithm of completion of $\Re_{0}$. Then $\Re^{+}$is a rewriting system for the right-angled Artin monoid $M$ defined by $\Im$.

Proof. We will show by induction on the number of steps in the KnuthBendix algorithm of completion of $\Re_{0}$ that at each step a positive rule is created by the overlap of two positive rules. At the 0 -th step, the set of positive rules is $\Re_{0}^{+}=\left\{x^{\gamma} y^{\epsilon} \rightarrow y^{\epsilon} x^{\gamma}\right.$, where $\gamma=\epsilon=1$, for each edge $x-y \in \Im\}$.

The rewriting system $\Re_{0}^{+}$is a rewriting system for the right-angled 
Artin monoid $M$ defined by $\Im$, since to each edge in $\Im$, there corresponds exactly one rule in $\Re_{0}^{+}$.

At the first step, we have overlaps between rules $x^{\gamma} y^{\epsilon} \rightarrow y^{\epsilon} x^{\gamma}$ and $y^{\epsilon} z^{\delta} \rightarrow z^{\delta} y^{\epsilon}$, where $\gamma, \delta, \epsilon= \pm 1$, and the new rule obtained is $x^{\gamma} z^{\delta} y^{\epsilon} \rightarrow$ $y^{\epsilon} x^{\gamma} z^{\delta}$. The rule $x^{\gamma} z^{\delta} y^{\epsilon} \rightarrow y^{\epsilon} x^{\gamma} z^{\delta}$ is a positive rule if and only $\gamma=$ $\delta=\epsilon=1$. Note that the words $x^{\gamma} z^{\delta} y^{\epsilon}$ and $y^{\epsilon} x^{\gamma} z^{\delta}$ are freely reduced, since otherwise $z=x$ and this would contradict the total ordering on the generators.

Assume that at the $(n-1)$-th step in the Knuth-Bendix algorithm of completion, all the positive rules were created by overlaps between positive rules. We will assume that at the $n$-th step, there is a positive rule which is created by an overlap between rules which are not positive.

Let $u x \rightarrow x u$ be a rule in $\Re_{n-1}$, which satisfies the condition on prefixes, where $u=t_{1} t_{2} . . t_{n-1} t_{n}$ and $x, t_{i} \in X \bigcup X^{-1}$.

Let $x y \rightarrow y x$ be a rule in $\Re_{0}$, where $y \in X \bigcup X^{-1}$, such that there is an overlap of $u x \rightarrow x u$ at left and $x y \rightarrow y x$ at right which creates a positive rule.

By proposition 4.9(ii), $\Re$ satisfies the following condition: one side of a rule in $\Re$ is a positive word if and only if the other side is a positive word. So, if $u x$ or $x y$ are not positive words, then the words $u y x$ and $x u y$ are not positive also and $u y x$ and $x u y$ reduce to positive words only if there are free reductions which eliminate the generators with negative exponent sign. So, $t_{n}=y^{-1}$ and we have:

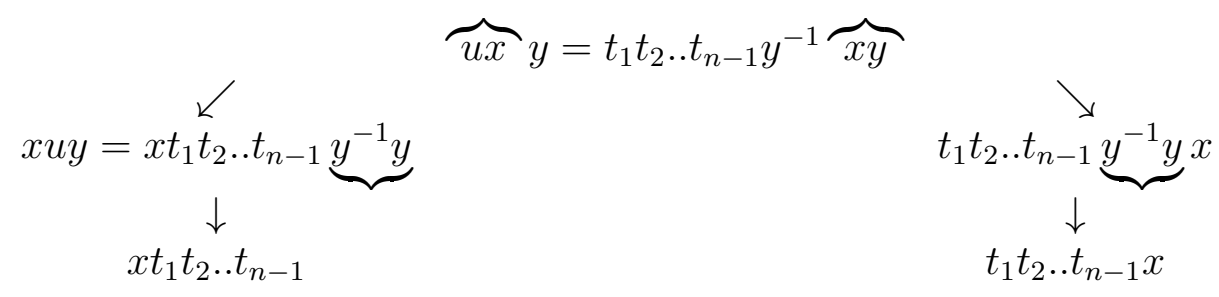

The word $t_{1} t_{2} . t_{n-1}$ belongs to $\operatorname{pref}(u)$ and the rule $u x \rightarrow x u$ satisfies the condition on prefixes, so from lemma 4.6, there is a rule $t_{1} t_{2} . . t_{n-1} x \rightarrow x t_{1} t_{2} . . t_{n-1}$ and from the condition (C) in proposition 4.9 it was obtained at the $(n-2)$-th step. So, a positive rule cannot be created by this overlap.

\section{Proof of theorem 4.1}

Proof. Let define a total ordering $<$ on the generators and their inverses and let $\Re_{0}=\left\{x^{\gamma} y^{\epsilon} \rightarrow y^{\epsilon} x^{\gamma}\right.$, where $\gamma$ and $\epsilon$ take all values \pm 1 , for each edge $x-y \in \Im\}$ be a rewriting system for the right angled Artin group defined by $\Im$, using the length-lexicographical ordering induced by $<$. Let $\Re$ be the complete rewriting system obtained from the Knuth-Bendix algorithm of completion applied on $\Re_{0}$. 
Then by proposition 4.9, $\Re$ satisfies the condition $C^{+}$and from proposition 4.10, $\Re$ satisfies the condition on $\Re^{+}$.

So, from theorem 3.9, the right angled Artin monoid embeds in the corresponding group.

In appendix B, we give a very simple proof of the embedding of right angled Artin monoids with complete defining graph or with defining graph, such that all the closed paths have even length, in their corresponding groups. In fact, we find in these two cases a finite and complete rewriting system (with no need of completion) which satisfies the condition $C^{+}$and the condition on $\Re^{+}$, by using some elementary tools from graph theory.

\section{Appendix A :PROOF OF LEMMA 4.6}

Before we give the proof, we need the following lemma and definition.

Lemma 5.1. Assume the set $\left\{t_{1}, t_{2}, . ., t_{k}\right\}$ is a clique. Then any word $w=t_{i_{1}} t_{i_{2}} . . t_{i_{k}}$, where $i_{j} \in\{1,2, . ., k\}$, can be reduced to its normal form modulo $\Re$ by using only rules from $\Re_{0}$. That is, no new rule is created from the overlaps between rules involving only elements from a clique.

Proof. Since there is a total ordering on the generators, each word $w=t_{i_{1}} t_{i_{2}} . . t_{i_{k}}$, where $i_{j} \in\{1,2, . ., k\}$ reduces to a unique irreducible word, which is the least one lexicographically and which is obtained by the application of rules from $\Re_{0}$.

Let $w$ be a word in the free group generated by $X$. Assume $w=$ $t_{1} t_{2} t_{3} \ldots t_{k}$, where $t_{i} \in X \bigcup X^{-1}$ for $1 \leq i \leq k$. Then, we say that $t_{1}, t_{2}, . ., t_{i-1}$ appear before $t_{i}$ and $t_{i+1}, t_{i+2}, . ., t_{k}$ appear after $t_{i}$.

Proof. We prove that by induction on the number of steps in the algorithm of completion and we prove also the following condition $(* *)$ : if $x$ is the last letter in the left-hand side of a rule and $x$ commutes with all the letters appearing before, then in the right-hand side $x$ is the first letter.

At the 0 -th step, the assumptions hold trivially. At the first step of completion, there is an overlap between $t_{1} x \rightarrow x t_{1}$ and $x t_{i_{2}} \rightarrow t_{i_{2}} x$ in $\Re_{0}, i_{2} \neq 1$, which gives

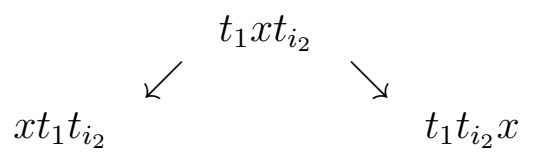

The rule $t_{1} t_{i_{2}} x \rightarrow x t_{1} t_{i_{2}}$ is obtained and assumption (**) holds. The word $x t_{1} t_{i_{2}}$ is irreducible modulo $\Re$ since it is a subword of $x t_{1} t_{i_{2}} . t_{i_{n}}$. 
The word $t_{1} t_{i_{2}} x$ is irreducible modulo $\Re_{0}$, since each subword is irreducible modulo $\Re_{0}$ : the word $t_{1} t_{i_{2}}$ is irreducible modulo $\Re_{0}$ since otherwise there would be an inclusion ambiguity with the rule $t_{1} t_{i_{2}} . . t_{i_{n}} x \rightarrow$ $x t_{1} t_{i_{2}} . . t_{i_{n}}$ in $\Re$ and this is not possible.

Assume that at the $(m-1)-t h$ step of completion of $\Re_{0}$, the rule $t_{1} t_{i_{2}} . t_{i_{n-2}} x \rightarrow x t_{1} t_{i_{2}} . . t_{i_{n-2}}$ is obtained, where $t_{i_{j}} \in\left\{t_{2}, . ., t_{k}\right\}$. Then at the $m-t h$ step, from the overlap between this rule and $x t_{i_{n-1}} \rightarrow t_{i_{n-1}} x$ $\left(i_{n-1} \neq 1\right)$, we have:

$$
\swarrow \overbrace{t_{1} t_{i_{2}} . . t_{i_{n-2}} x} t_{i_{n-1}} \searrow t_{t_{1} t_{i_{2}} . . t_{i_{n-2}} t_{i_{n-1}} x}
$$

We need to show that a new rule is created, with the word $x t_{1} t_{i_{2} .} . t_{i_{n-2}} t_{i_{n-1}}$ in the right-hand side and the word $t_{1} t_{i_{2}} . . t_{i_{n-2}} t_{i_{n-1}} x$ in the left-hand side. To do that, we need to show that both these words are irreducible modulo the rewriting system $\Re_{m-1}$ obtained one step before. In fact, the word $x t_{1} t_{i_{2}} . t_{i_{n-2}} t_{i_{n-1}}$ is irreducible modulo $\Re$ since it is a subword of $x t_{1} t_{i_{2}} . . t_{i_{n}}$, which is an irreducible word modulo $\Re$. We show that the word $t_{1} t_{i_{2}} . . t_{i_{n-2}} t_{i_{n-1}} x$ is irreducible modulo $\Re_{m-1}$, by showing that each of its subword cannot occur as a left-hand side of a rule in $\Re_{m-1}$. If there is a rule $t_{i_{j}} t_{i_{j+1}} . . t_{i_{n-1}} x \rightarrow \ldots$, where $i_{j} \neq 1$. Then from assumption $(* *)$, we have that $t_{i_{j}} t_{i_{j+1}} . . t_{i_{n-1}} x \rightarrow x .$. , since $x$ commutes with all the $t_{i_{j}}$ and this would imply that $t_{i_{j}}>x$. But from the existence of the rule $x t_{i_{j}} \rightarrow t_{i_{j}} x$ in $\Re_{0}$, it holds that $x>t_{i_{j}}$. So, there can be no such rule and it is easy to check that for the other subwords, so the word $t_{1} t_{i_{2}} . t_{i_{n-2}} t_{i_{n-1}} x$ is irreducible modulo $\Re_{m-1}$.

So, there is the rule $t_{1} t_{i_{2}} . . t_{i_{n-2}} t_{i_{n-1}} x \rightarrow x t_{1} t_{i_{2}} . t_{i_{n-2}} t_{i_{n-1}}$ in $\Re$ and assumption $(* *)$ holds.

From the definition of $\Re_{0}$, if there are rules $t_{1} x \rightarrow x t_{1}$ and $x t_{i} \rightarrow t_{i} x$ for $2 \leq i \leq k$, then there are also the rules $t_{1} x^{-1} \rightarrow x^{-1} t_{1}$ and $x^{-1} t_{i} \rightarrow t_{i} x^{-1}$ for $2 \leq i \leq k$. So, in the same process as before the rule $u x^{-1} \rightarrow x^{-1} u$ is created.

\section{Appendix B}

6.1. Right angled Artin groups with defining graph such that all the closed paths have even length. Let $\Im$ be the defining graph of a right-angled Artin group $G$ and the corresponding right-angled Artin monoid $M$, such that all the closed paths in $\Im$ have even length. As before, we denote by $X$ the set of vertices in $\Im$ or equivalently the set of generators of $G$ and by $X^{-1}$ the set of inverses of the elements in $X$. We will show that $G$ admits a finite and complete rewriting system $\Re$ satisfying the condition $C^{+}$by using some very simple tools from graph theory (see [15]). In fact, we will define a total ordering ' $>$ ' on $X \bigcup X^{-1}$ such that the rewriting system 
$\Re=\left\{x_{i}^{\gamma} x_{j}^{\epsilon} \rightarrow x_{j}^{\epsilon} x_{i}^{\gamma}\right.$, where $x_{i}^{\prime}>^{\prime} x_{j}$ and $\gamma, \epsilon$ take all values \pm 1$\}$ is finite and complete with no need of completion.

Lemma 6.1. Let $\Im$ be the defining graph of a right-angled Artin group such that all the closed paths have even length. Then the vertices of $\Im$ can be coloured in two colours (black and white) such that any two adjacent vertices (i.e vertices connected by an edge) have different colours.

Proof. The graph $\Im$ is simple and satisfies the condition that all the closed paths have even length, so using the proposition [15, p.81] the vertices of $\Im$ can be coloured in two colours: black and white.

Assume a colouring is assigned to $\Im$. Denote by $B$ the set of generators corresponding to vertices which are coloured in black in $\Im$ and by $W$ the set of generators corresponding to vertices which are coloured in white in $\Im$. By definition of the colouring, $B \cap W=\emptyset$ and $X=B \bigcup W$. We denote by $B^{-1}$ the set of inverses of the elements in $B$ and by $W^{-1}$ the set of inverses of the elements in $W$.

Lemma 6.2. The colouring of the vertices in $\Im$ defines a well-founded, total ordering on $X \bigcup X^{-1}$.

Proof. We order arbitrarily the elements in $B \cup B^{-1}$ and also the elements in $W \cup W^{-1}$, but we require that the least element from $B \cup B^{-1}$ is greater than the greatest element from $W \cup W^{-1}$. This ordering is well-founded, i.e there can be no infinite sequence

$x_{1}>x_{2}>\ldots>x_{k}>\ldots$, since $\left(B \cup B^{-1}\right) \bigcap\left(W \cup W^{-1}\right)=\emptyset$ and it is total since $X \bigcup X^{-1}=(B \bigcup W) \bigcup\left(B^{-1} \bigcup W^{-1}\right)$.

Remark 6.3. From the proof of lemma6.2, it results for each edge $a-b$ that the corresponding rules are oriented $a^{\gamma} b^{\epsilon} \rightarrow b^{\epsilon} a^{\gamma}$ (where $\gamma$ and $\epsilon$ take all values \pm 1 ) if and only if $a$ is coloured in black and $b$ in white.

Proposition 6.4. Let $>_{\text {lex }}$ be the length-lexicographical ordering induced by the total ordering on $X \bigcup X^{-1}$ defined above and let $\Re$ be the rewriting system obtained using $>_{\text {lex }}$. Then $\Re$ is finite and complete and it satisfies the condition $C^{+}$. Further, $\Re^{+}$is a rewriting system for $M$.

Proof. $\Re$ is terminating since it is defined by a length-lexicographical ordering, it is finite and it satisfies $C^{+}$from lemma 4.3, So, it remains to show that during the Knuth-Bendix algorithm of completion, the overlaps of the rules resolve with no need to add new rules. In fact, we will show that there are no overlaps between the rules themselves but only between the rules and the rules of kind $x x^{-1} \rightarrow 1$ and $x^{-1} x \rightarrow 1$ $(x \in X)$ and these resolve from lemma 4.3. Assume there is an overlap between two rules $x^{\gamma} y^{\epsilon} \rightarrow y^{\epsilon} x^{\gamma}$ and $y^{\epsilon} z^{\delta} \rightarrow z^{\delta} y^{\epsilon}$, where $x, y, z \in X$ and $\gamma, \delta, \epsilon= \pm 1$. If there is a rule $x^{\gamma} y^{\epsilon} \rightarrow y^{\epsilon} x^{\gamma}$ in $\Re$, then it means that 
there is an edge $x-y$ in $\Im$ with $x$ coloured in black and $y$ coloured in white. In the same way, if there is a rule $y^{\epsilon} z^{\delta} \rightarrow z^{\delta} y^{\epsilon}$ in $\Re$, then it means that there is an edge $y-z$ in $\Im$ with $y$ coloured in black and $z$ coloured in white. But this is a contradiction to the colouring of $\Im$.

\subsection{Right angled Artin groups with a complete defining graph.}

Let $M$ be the free commutative monoid on $n$ generators with the standard presentation $M=\left\langle x_{1}, . ., x_{n}\right| x_{i} x_{j}=x_{j} x_{i}, n \geq i>j \geq 1>$. The left and right graphs $\Gamma_{l}$ and $\Gamma_{r}$ both have cycles, so nothing can be concluded here using Adian's criteria.

Yet, it is well known that this monoid embeds in the free abelian group on $n$ generators or in other words in the right angled Artin group with a complete defining graph on $n$ vertices. We show this well-known result, using the criteria of section 3 . Indeed, we give a complete and finite rewriting system which satisfies the condition $C^{+}$.

Let $\left\langle x_{1}, . ., x_{n}, x_{1}^{-1}, . ., x_{n}^{-1}\right| x_{i} x_{j}=x_{j} x_{i}, n \geq i>j \geq 1>, x_{i}^{-1} x_{i}=$ $\left.x_{i} x_{i}^{-1}=1,1 \leq i \leq n\right\rangle$ be the monoid presentation of the free abelian group on $n$ generators. Let $\Re$ be the rewriting system defined for the presentation above, using a length-lexicographical ordering induced by the following total ordering on the generators: $x_{n}>x_{n}^{-1}>\ldots>x_{2}>$ $x_{2}^{-1}>x_{1}>x_{1}^{-1}$. So, $\Re$ can be described as the following set of rules: $\Re=\left\{x_{i}^{\gamma} x_{j}^{\epsilon} \rightarrow x_{j}^{\epsilon} x_{i}^{\gamma}\right.$, where $n \geq i>j \geq 1$ and $\left.\gamma, \epsilon= \pm 1\right\}$ This rewriting system is terminating since it is defined by a length-lexicographical ordering and one can check that the overlaps between the rules which occur resolve with no need to add new relations. So $\Re$ is finite and complete and it satisfies the condition $C^{+}$and the condition on $\Re^{+}$. By theorem 3.9, the monoid embeds in the group.

\section{REFERENCES}

[1] S.I.Adian, Defining relations and algorithmic problems for groups and semigroups, Proc. Steklov Inst.Math.85(1967), p.1-152.

[2] R.V. Book and F. Otto, String-Rewriting systems, Springer-Verlag, 1993.

[3] R.Charney, Injectivity of the positive monoid for some infinite type Artin groups, Geometric group theory down under (Canberra, 1996), p.103-118, de Gruyter, Berlin, 1999.

[4] J.R. Cho and S.Pride, Embedding semigroups into groups, and the asphericity of semigroups, Internat. J. Algebra Comput.3(1993),no. 1,p.1-13.

[5] D.E. Cohen, String Rewriting-A Survey for Group Theorists, in Geometric Group Theory, vol.1, G.A.Niblo and M.A.Roller (eds), Lecture Notes of the London .Math.Soc.181 (1993).

[6] S.M.Hermiller and J.Meier, Algorithms and geometry for graph products of groups, J. Algebra 171 (1995),no. 1, p.230-257.

[7] G.Huet, A complete proof of correctness of the Knuth Bendix completion algorithm, J. of computer and system sciences 23, p.11-21 (1981).

[8] E.V.Kashintsev, Small cancellation conditions and embeddability of semigroups in groups, Internat.J.Algebra Comput.2 (1992), no. 4, p.433-441. 
[9] S.Krstic, Embedding semigroups in groups: a geometrical approach, Publications de l'Institut Mathematique, nouvelle serie,tome38(52),1985,p.69-82.

[10] P.Le Chenadec, Canonical forms in finitely presented algebras, Pitman, London, 1986.

[11] L.Paris, Artin monoids inject in their groups, Comment.Math.Helv 77 (2002),n.3, p.609-637.

[12] J.Pederson and M.Yoder, Term rewriting for the conjugacy problem and the braid groups, J.Symbolic Comput.18(1994), 563-572.

[13] C.C.Sims, Computing with finitely presented groups, Wiley, 1996.

[14] L.Van Wyk, Graph groups are biautomatic, J. Pure Appl. Algebra 94 (1994), no. 3, 341-352.

[15] R.J.Wilson, Introduction to Graph Theory, Oliver and Boyd, Edinburgh,1972. 